\title{
UTILIZAÇÃO DE ENERGIA ELÉTRICA EM DIFERENTES SISTEMAS DE AQUECIMENTO PARA LEITÕES DESMAMADOS
}

\author{
JULIANA SARUBBI ${ }^{1}$, LUIZ A. ROSSI ${ }^{2}$, DANIELLA J. DE MOURA ${ }^{3}$, \\ RAFAEL A. DE OLIVEIRA ${ }^{4}$, EDUARDO DAVID ${ }^{5}$
}

\begin{abstract}
RESUMO: A eficiência do uso de energia elétrica no aquecimento de leitões em fase de creche e maternidade pode ser viabilizada sem prejudicar o conforto térmico dos suínos. Neste trabalho, foram comparadas três tecnologias de aquecimento de leitões em fase de creche, quanto ao uso de energia elétrica e ao ambiente térmico. Estudaram-se três tratamentos: resistências elétricas suspensas (TR), piso aquecido (TP) e aquecimento por convecção (TA). Os critérios de comparação foram: consumo de energia elétrica $(\mathrm{kWh})$, demanda máxima de potência $(\mathrm{kW})$, consumo específico $(\mathrm{kWh} / \mathrm{kg}$ de suíno vivo produzido), a quantidade de energia elétrica consumida $(\mathrm{kWh})$ para o aumento de $1^{\circ} \mathrm{C}$ em $1 \mathrm{~m}^{3}$ de ar, a temperatura de bulbo seco $\left({ }^{\circ} \mathrm{C}\right)$ e o Índice de Temperatura e Umidade (ITU). O sistema de aquecimento por piso (TP) foi melhor sob o aspecto de uso de energia elétrica, e o sistema de aquecimento por resistências elétricas suspensas (TR) foi o que melhor atendeu às necessidades térmicas dos animais.
\end{abstract}

PALAVRAS-CHAVE: suinocultura, creche, racionalização de energia elétrica, piso aquecido.

\section{ELECTRICAL ENERGY USE IN DIFFERENT HEATING SYSTEMS FOR WEANED PIGLETS}

\begin{abstract}
Heating piglets in nursery and farrowing may be improved to save energy, without affecting the animals' welfare. The objective of this research was to compare the electrical energy use and thermal environment promoted by three heating technologies for piglets in nursery. The heating technologies studied were: TR - suspended electrical resistors, TP - heated floor and TA convection heating. Electrical energy consumption $(\mathrm{kWh})$, maximum power demand requirements $(\mathrm{kW})$, specific consumption $(\mathrm{kWh} / \mathrm{kg}$ of produced live body weight), efficiency of heating system $\left({ }^{\circ} \mathrm{C} / \mathrm{m}^{3}\right.$ air) and dry-bulb temperature $\left({ }^{\circ} \mathrm{C}\right)$ and Temperature-humidity Index (THI) were evaluated. The heated floor system was better under the aspect of electrical energy use. The electrical resistance heating system was the best regarding to the animals' thermal comfort needs.
\end{abstract}

KEYWORDS: swine production, pig nursery, energy saving, heated floor.

\section{INTRODUÇÃO}

Para atender às demandas de quantidade e qualidade de proteína animal, o produtor necessita investir em novas tecnologias para incluir-se no mercado pecuário atual, que está mais competitivo. A manutenção da competitividade na produção de suínos depende, principalmente, da qualidade do produto e da redução do custo de produção do suíno vivo.

Para melhorar a qualidade do produto, devem ser buscadas características comumente consideradas, como carcaças de melhor qualidade (menor espessura de toucinho, carne magra, entre outros), mas também novas demandas de mercado, como é o caso de animais criados sob condições de bem-estar e sustentabilidade.

\footnotetext{
${ }^{1}$ Profa. Dra., Departamento de Zootecnia, Universidade Federal de Santa Maria, CESNORS, Palmeira das Missões - RS, Pesquisadora colaboradora FEAGRI/UNICAMP, jusarubbi@ hotmail.com.

${ }^{2}$ FEAGRI/UNICAMP, Cidade Universitária Zeferino Vaz s/n, Campinas - SP, rossi@agr.unicamp.br.

${ }^{3}$ FEAGRI/UNICAMP, Cidade Universitária Zeferino Vaz s/n, Campinas - SP, daniella.moura@agr.unicamp.br.

${ }^{4}$ Prof Dr., FEAGRI/UNICAMP, Cidade Universitária Zeferino Vaz s/n, Campinas - SP, augustus@agr.unicamp.br.

${ }^{5}$ Doutorando, FEAGRI/UNICAMP, Cidade Universitária Zeferino Vaz s/n, Campinas - SP, eduardo.david@agr.unicamp.br

Recebido pelo Conselho Editorial em: 16-9-2009

Aprovado pelo Conselho Editorial em: 5-10-2010
} 
O uso racional de energia na suinocultura é importante para a sustentabilidade da atividade, pois a correta utilização da energia elétrica pode contribuir para a viabilidade econômica da criação, além de contribuir para a viabilidade ambiental e para o bem-estar dos animais.

Segundo a Companhia Nacional de Abastecimento (CONAB), o custo da energia elétrica e de outros combustíveis utilizados na produção de suínos, nos Estados brasileiros, no mês de novembro de 2008, variou de 0,4 a 2,6\% do custo total de produção (CONAB, 2008). Os dados apresentados são da energia elétrica, juntamente com outras fontes de energia utilizadas na produção de suínos e há discrepância da porcentagem de utilização destas fontes na produção de suínos entre os Estados. Neste caso, não há dados específicos para o uso de energia elétrica.

Não são abundantes literaturas atuais e específicas sobre energia elétrica na suinocultura. De acordo com GIROTTO \& TALAMINI (1998), o consumo médio da energia elétrica por suíno terminado no Brasil, no ano de 1994, era de 2,03 $\mathrm{kWh}$ nimal $^{-1}$ terminado. TALAMINI et al. (2006) estimaram que o consumo de uma granja de suínos é de $150 \mathrm{kWh}$ por matriz alojada.

O consumo de energia elétrica pode ser reduzido em uma granja de suínos, com algumas medidas, como, por exemplo, a utilização de um termostato simples. O termostato pode ser interessante no uso racional de energia elétrica, permitindo corrigir as deficiências de manejo da fonte de calor e possibilitando a manutenção de um ambiente sempre estável, com economia de energia de 30 a 50\% em relação ao sistema que não o utiliza (BONETT \& MONTICELLI, 1997).

O aquecimento de leitões em fase de creche e maternidade é um setor da granja que consome muita energia elétrica. Desta forma, é necessário aumentar a eficiência no uso da energia elétrica sem prejudicar o bem-estar dos suínos.

XIN et al. (1997) compararam diferentes lâmpadas para aquecimento de leitões em maternidade e concluíram que a utilização de lâmpadas radiantes economizadoras de $175 \mathrm{~W}$ (Phillips PAR) ao invés de lâmpadas infravermelhas de $250 \mathrm{~W}$, foram capazes de proporcionar uma economia de 1,03 kWh/lâmpada/dia. Esta economia significou o equivalente a US $\$ 5,400$ ano $^{-1}$, para uma granja com 1.000 fêmeas, considerando-se as condições econômicas e de custos do mercado dos Estados Unidos (EUA). A lâmpada economizadora apresentou maior durabilidade e possibilitou a redução da mortalidade e maior ganho de peso dos leitões.

BESHADA et al. (2006), ao estudarem tapetes aquecidos e lâmpadas para o aquecimento de leitões em maternidade, não encontraram diferença significativa entre o ganho de peso e a mortalidade dos animais submetidos a estas duas tecnologias, mas relataram uma economia de $2,8 \mathrm{kWh}$ por leitegada.

Em virtude da grande quantidade de doenças, problemas comportamentais e agentes estressores comprovados que acometem suínos em fase de creche (MERLOT et al., 2004), existe a necessidade do desenvolvimento de trabalhos que possibilitem a minimização do estresse, principalmente térmico, destes animais.

Este trabalho teve o objetivo de comparar sistemas de aquecimento (resistências elétricas suspensas, piso aquecido e aquecimento por convecção) para leitões em fase de creche, sob os aspectos do conforto dos animais, uso de energia elétrica e de eficiência energética nos sistemas.

\section{MATERIAL E MÉTODOS}

Os experimentos foram realizados em uma granja suinícola, localizada em Boituva, Estado de São Paulo. A unidade de produção possuía criação intensiva confinada, ciclo completo e organização semanal da produção. Abrigava 1.000 matrizes suínas e produzia matrizes, provenientes de avós de uma empresa de genética mundialmente reconhecida.

Em cada uma das análises, um lote de 450 leitões desmamados (idade ajustada: 19 dias) foi distribuído em três baias idênticas. Cada tratamento foi alocado em uma baia com 150 animais, cujo piso possuía $1 / 3$ da área de concreto e $2 / 3$ de piso plástico vazado. A densidade utilizada foi de 
5 leitões $\mathrm{m}^{-2}$. Embora alguns autores afirmem que grandes grupos e altas densidades sejam capazes de reduzir o desempenho dos leitões (WOLTER et al., 2000), foram respeitadas as condições de manejo, alojamento e instalações já utilizadas na granja.

As salas que abrigavam as baias do estudo eram de alvenaria, cobertas com telhas de barro. Todas as baias eram suspensas e possuíam aproximadamente $26 \mathrm{~m}^{2}$. Cada baia foi coberta com forro polipropileno amarelo, alocado a $1,85 \mathrm{~m}^{2}$ do piso, visando a reduzir a área a ser aquecida. As salas possuíam janelas teladas, situadas nos dois lados, no sentido da largura, protegidas por cortinas de polipropileno amarelo. $\mathrm{O}$ manejo das cortinas foi realizado pelo funcionário, que abria as cortinas no período da manhã e as fechava à tarde.

Os leitões foram submetidos a três diferentes tratamentos que se diferenciaram pelos sistemas de aquecimento. $\mathrm{O}$ aquecimento foi realizado durante 14 dias. Foram realizados dois estudos observacionais, nos meses de junho e julho de 2008 (Análise 1 e Análise 2).

Os tratamentos comparados foram os seguintes:

TR (Resistências elétricas suspensas) - composto por 15 resistências elétricas de 200W, uniformemente distribuídas, suspensas, distando $1 \mathrm{~m}$ do piso da baia (Figura 1). O sistema foi controlado por um termostato digital simples (marca COEL, modelo HW4200) com o objetivo de aquecer os leitões por radiação. O sistema foi projetado para a manutenção da temperatura do ar entre $28-30{ }^{\circ} \mathrm{C}$. A potência instalada para este sistema foi de $3.000 \mathrm{~W}$.

TP (Piso aquecido) - composto por uma área de $10,4 \mathrm{~m}^{2}$, correspondente a $40 \%$ da área total da baia (Figura 1). A metade superior do piso foi feita de argamassa de cimento (Portland CP V ARI) e areia. A metade inferior foi feita de argamassa de cimento, areia e casca de arroz (material para isolamento), segundo recomendações de ROSSI et al. (2005). A temperatura do piso foi controlada eletronicamente por um sistema desenvolvido e construído para este fim, que manteve a temperatura do piso entre $28-30{ }^{\circ} \mathrm{C}$. Este sistema teve como objetivo fornecer calor ao leitão por condução. O piso foi mantido nesta temperatura após teste prévio de preferência e observação do comportamento dos leitões. Para o dimensionamento da resistência elétrica, foi utilizada a metodologia descrita por INCROPERA \& DEWITT (1998), baseada na Lei de Conservação de Energia. A potência instalada considerada para este sistema foi de $2.400 \mathrm{~W}$.

TA (Sistema de aquecimento por convecção) - composto por um intercambiador comercial de calor, que conduzia ar quente, proveniente das resistências elétricas, para o interior das salas. Este procedimento foi realizado utilizando-se de um ventilador. $\mathrm{O}$ ar foi distribuído por meio de uma tubulação de PVC de $15 \mathrm{~cm}$ de diâmetro e $7 \mathrm{~m}$ de comprimento, com três saídas de ar equidistantes, de modo que o ar fosse insuflado a partir do meio da instalação (Figura 1). O sistema foi controlado por um termostato digital simples (COEL- HW4200) que atuava de acordo com a temperatura do ar do interior das instalações. A tecnologia utilizou-se do princípio de transferência de calor por convecção. O sistema foi projetado para a manutenção da temperatura do ar entre 28 a $30{ }^{\circ} \mathrm{C}$. A potência total instalada para este sistema (ventilador + resistências elétricas) foi de aproximadamente $8.830 \mathrm{~W}$.

Em virtude da grande divergência encontrada na literatura quanto à temperatura do ar a que os leitões devem ser expostos aos 21 dias, mantiveram-se os leitões na zona de temperatura já utilizada pela granja, de $28^{\circ} \mathrm{C}$ e $30^{\circ} \mathrm{C}$. O critério de eleição da faixa de temperatura somou-se à recomendação de BROWN-BRANDL et al. (2003), apresentada na eq.(1):

$$
\text { Tideal }=0,0015 \mathrm{wt}^{2}-0,2969 \mathrm{wt}+30,537
$$

em que,

$\mathrm{T}_{\text {ideal }}$ - temperatura ambiente ideal para o animal, ${ }^{\circ} \mathrm{C}$, e wt - peso, kg. 


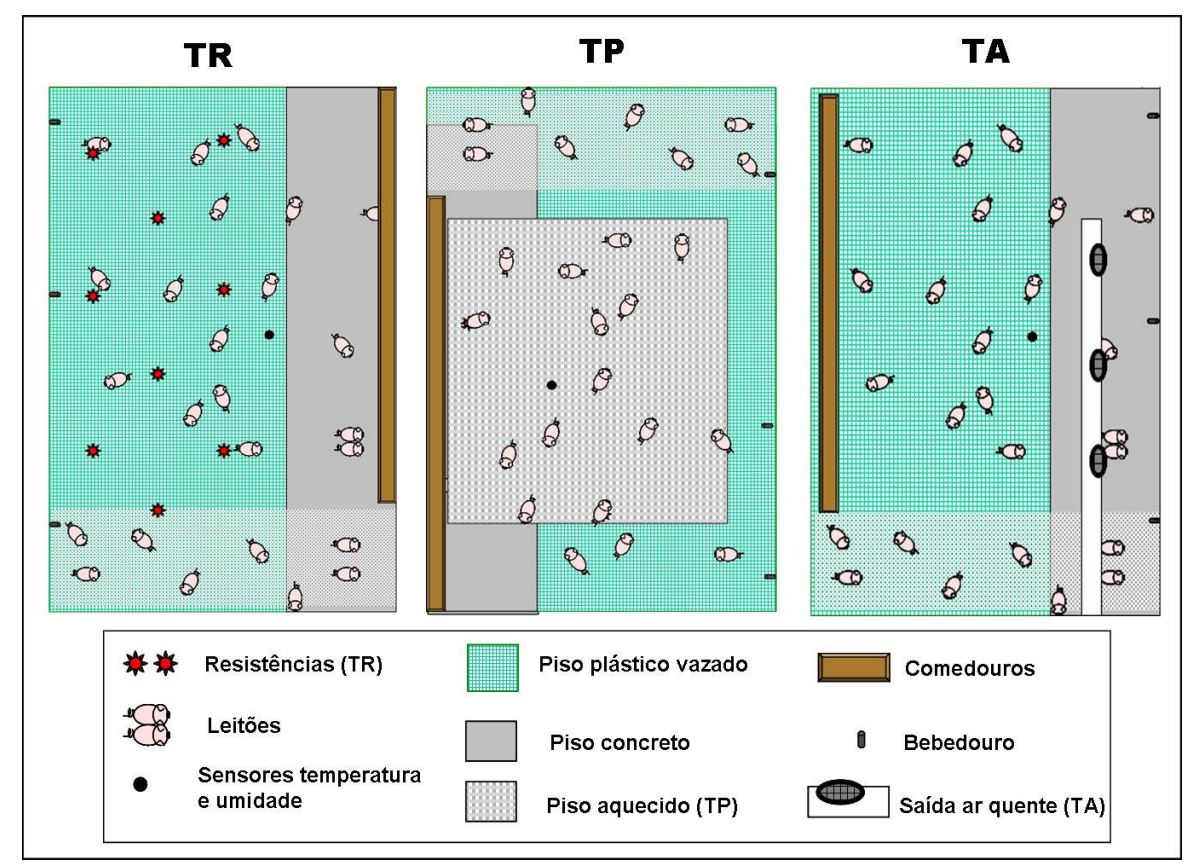

FIGURA 1. Desenho esquemático dos tratamentos. Layout of treatments.

A utilização de energia elétrica dos equipamentos foi avaliada através de medidores eletrônicos individuais (Embrasul, modelo RE6081), instalados nas caixas de distribuição de circuitos. Estes medidores foram acoplados a outro acumulador ou registrador de dados (datalogger) específico. Os registros das variáveis elétricas foram realizados a cada 15 minutos, 24 horas por dia, durante os experimentos. Os dados dos medidores foram analisados em um programa computacional específico para esta finalidade.

As variáveis elétricas consideradas para a comparação dos tratamentos foram a demanda máxima de potência $(\mathrm{kW})$ e o consumo de energia elétrica $(\mathrm{kWh})$. A demanda máxima registrada indica o maior valor de demanda de energia elétrica registrado no período, sendo que a demanda é o consumo de energia dividido pelo tempo de medição, no período considerado, e é dada em kW.

Todos os parâmetros foram considerados para o sistema com todos os seus componentes eletrônicos, incluindo componentes eletrônicos, leds, ventiladores para arrefecimento dos controladores (coolers), etc.

A variável objetiva usada para avaliar o desempenho produtivo dos animais foi o ganho de peso do grupo no período estudado. Os animais foram pesados em grupos de aproximadamente 10 animais, e os pesos dos grupos foram somados dentro de cada tratamento. O ganho de peso foi considerado para o cálculo do consumo específico (eq.(2)).

Para avaliar a eficiência energética dos tratamentos, foi utilizado o consumo específico. Realizou-se a análise do consumo de energia $(\mathrm{kWh})$ em relação ao produto gerado (kg de peso vivo na saída da creche). Esta relação é dada pela eq.(2):

$$
\mathrm{Ces}=\frac{\mathrm{CAi}}{\mathrm{QPi}}
$$

em que,

Ces - consumo específico, em $\mathrm{kWh} / \mathrm{kg}$ de suíno vivo produzido;

$\mathrm{CAi}$ - consumo de energia elétrica pelo lote, $\mathrm{kWh}$;

QPi - quantidade de produto produzido no lote pela unidade consumidora (baia), kg suíno vivo, e

i - índice referente ao lote de criação de suínos obtido através de análise histórica dos dados. 
Ainda para a avaliação da eficiência energética, OLIVEIRA et al. (2006) determinaram o consumo de energia por metro quadrado de solo, em um estudo sobre casa de vegetação aquecida. Neste trabalho, foi calculada a quantidade de energia elétrica consumida para o aumento de $1{ }^{\circ} \mathrm{C} \mathrm{em}$ $1 \mathrm{~m}^{3}$ de ar, nesta edificação, buscando avaliar a eficiência dos sistemas de aquecimento. Este cálculo foi realizado tomando como base a diferença entre as temperaturas no exterior e no interior das instalações.

Para o acompanhamento da temperatura de bulbo seco (Tbs) dos ambientes externo e internos, indicadores desta variável (modelo UMMI da marca BethaEletronica ${ }^{\circledR}$ ) foram colocados no centro das salas.

Cada indicador possui um registrador e acumulador de dados (datalogger) que possibilita que os dados sejam transferidos para o computador, visando a posterior análise. Por meio deste sistema, foram obtidos e acumulados dados de temperatura a cada 15 minutos. Com estes dados, foi calculado o Índice de Temperatura e Umidade (ITU), com as adaptações recomendadas por SARUBBI (2009).

Para comparação entre os tratamentos, após a verificação da normalidade dos dados, para Tbs, realizaram-se Análise de Variância e Teste de Médias (Tukey) $(\mathrm{p}<0,05)$. Os dados de energia elétrica foram avaliados pelo teste não paramétrico de Kruskal-Wallis $(\mathrm{p}<0,05)$.

\section{RESULTADOS E DISCUSSÃO}

O consumo de energia elétrica é o registro do quanto de energia elétrica foi consumida durante um determinado tempo e, por isso, expresso em $\mathrm{kWh}$. Na Tabela 1, estão apresentados os valores de consumo médio diário de energia elétrica, em $\mathrm{kWh}$, para cada um dos tratamentos, nos períodos estudados.

TABELA 1. Consumo total de energia elétrica por diferentes sistemas de aquecimentos para leitões em creche, com 2 semanas de uso. Total electric energy consumption for different heating systems for piglet nursery, during two weeks of use.

\begin{tabular}{cccc}
\hline & \multicolumn{3}{c}{ Consumo de Energia Elétrica $(\mathrm{kWh})$} \\
\cline { 2 - 4 } & $\mathrm{TR}$ & $\mathrm{TP}$ & TA \\
\hline Análise 1 & $69,16^{\mathrm{b}}$ & $41,62^{\mathrm{a}}$ & $200,24^{\mathrm{c}}$ \\
Análise 2 & $115,32^{\mathrm{b}}$ & $44,8^{\mathrm{a}}$ & $278,14^{\mathrm{c}}$ \\
\hline
\end{tabular}

TR - resistências elétricas; TP - piso aquecido; TA - sistema de aquecimento por convecção.

O aquecimento por convecção foi o que mais consumiu energia elétrica, sendo, portanto, o pior dos tratamentos sob este aspecto, visto que o aumento do consumo de energia elétrica aumenta o custo de operação do sistema.

O aquecimento por piso aquecido foi o melhor porque consumiu menos energia elétrica. XIN \& ZHANG (1999) determinaram que a utilização de tapete aquecido pode oferecer economia de 40 a 60\%, quando confrontado com a utilização de lâmpadas radiantes (inclusive economizadoras). Estes dados são semelhantes aos encontrados nestas análises, quando se considera a comparação com as resistências elétricas.

No caso da comparação com o piso aquecido com as resistências, houve economia de, aproximadamente, $80 \%$. As médias de todos os dados de energia elétrica foram diferentes entre os tratamentos quando realizado o teste não paramétrico de Kruskal-Wallis $(\mathrm{p}<0,05)$, nas duas análises, conforme apresenta a Tabela 2. 
TABELA 2. Média do consumo de energia elétrica nos períodos estudados. Mean of electrical energy consumption during the studied periods.

\begin{tabular}{cccc}
\hline & \multicolumn{4}{c}{ Média de Consumo de Energia Elétrica $(\mathrm{kWh})$} \\
\cline { 2 - 4 } & TR & TP & TA \\
\hline Análise 1 & $2,88^{\mathrm{b}}$ & $0,44^{\mathrm{a}}$ & $8,34^{\mathrm{c}}$ \\
Análise 2 & $2,92^{\mathrm{b}}$ & $0,39^{\mathrm{a}}$ & $7,71^{\mathrm{c}}$ \\
\hline
\end{tabular}

Médias seguidas de letras diferentes na mesma linha diferem $(\mathrm{p}<0,05)$ pelo teste de Kruskal-Wallis. TR - resistências elétricas; TP - piso aquecido; TA - sistema de aquecimento por convecção.

A demanda máxima de energia elétrica registrada indica o maior valor de demanda de energia elétrica registrado no período. Os dados relativos à demanda máxima registrada, para cada tratamento e em cada período, estão apresentados na Tabela 3.

TABELA 3. Demanda máxima registrada nos diferentes sistemas de aquecimento para leitões em creche. Maximum demand registered in different heating systems for piglet nursery.

\begin{tabular}{cccc}
\hline & \multicolumn{3}{c}{ Demanda Máxima Registrada $(\mathrm{kW})$} \\
\hline & TR & TP & TA \\
\cline { 2 - 4 } Análise 1 & 2,99 & 1,36 & 8,71 \\
Análise 2 & 3,14 & 0,88 & 8,56 \\
\hline
\end{tabular}

TR - resistências elétricas; TP - piso aquecido; TA - sistema de aquecimento por convecção.

A maior demanda máxima de energia elétrica registrada, de 8,71 e $8,56 \mathrm{~kW}$, nas análises 1 e 2 , respectivamente, foi registrada no tratamento TA. Isso se explica em virtude da maior carga instalada neste sistema. Com a utilização do TP, foram observadas menores demandas máximas de energia, de 1,36 e 0,88 kW, nas análises 1 e 2, respectivamente, com a utilização do controlador e nas condições de temperatura avaliadas (Tabela 7).

Considerando a análise 1, a demanda máxima de TP foi igual a 15,61\% da TA. Já TR apresentou demanda máxima igual a $34,33 \%$ da registrada para TA. Durante a análise 2, TP e TR apresentaram demanda máxima registrada 10,28 e 36,68\%, respectivamente, em relação à obtida para TA.

No dimensionamento do piso aquecido, verificou-se a necessidade de $2,4 \mathrm{~kW}$ de potência instalada para atingir $30^{\circ} \mathrm{C}$ de temperatura na superfície do piso e há possibilidades para a melhoria do projeto do piso aquecido, em relação à eficiência energética.

Considerou-se o ganho de peso total no período estudado para o cálculo dos consumos específicos de energia elétrica dos sistemas. Na Tabela 4, estão apresentados resultados de ganho de peso total de cada lote ao final das análises observacionais 1 e 2.

TABELA 4. Ganho de peso total do lote, em $\mathrm{kg}$ de peso vivo, de leitões submetidos a diferentes sistemas de aquecimento na creche. Total gained body mass of piglets submitted to different heating systems in nursery.

\begin{tabular}{cccr}
\hline & \multicolumn{3}{c}{ Ganho de Peso Total do Lote $(\mathrm{kg})$} \\
\cline { 2 - 4 } & TR & TP & TA \\
\hline Análise 1 & 526,5 & 732,0 & 924,0 \\
Análise 2 & 844,2 & 750,3 & $1.081,2$ \\
\hline
\end{tabular}

TR - resistências elétricas; TP - piso aquecido; TA - sistema de aquecimento por convecção.

BESHADA et al. (2006) não encontraram diferenças no ganho de peso dos leitões, em um estudo feito com leitões de maternidade, utilizando diferentes sistemas de aquecimento. Neste estudo, com a utilização do tratamento TA, foi possível produzir a maior quantidade absoluta de 
produto ( $\mathrm{kg}$ de peso vivo). Mas a comparação da eficiência dos sistemas foi realizada por meio da análise do consumo específico (Ces). Isto porque, produzir mais produto, só é compensatório se o custo de produção não aumentar substancialmente, de modo a inviabilizar a produção.

O Ces, neste caso, avalia a energia elétrica consumida para a produção de $1 \mathrm{~kg}$ de peso vivo de suíno. Na Tabela 5, estão expostos os valores de Ces para cada sistema estudado, nas duas análises.

TABELA 5. Consumo específico (Ces) de diferentes sistemas de aquecimento para creche de leitões, em $\mathrm{kWh} \mathrm{kg}^{-1}$ de peso vivo de suíno produzido. Mean dry- bulb temperature $\left({ }^{\circ} \mathrm{C}\right)$ and THI values in piglet nursery for different heating systems.

\begin{tabular}{crcc}
\hline & \multicolumn{3}{c}{ Consumo Específico $\left(\mathrm{kWh} \mathrm{kg}^{-1}\right)$} \\
\cline { 2 - 4 } & TR & TP & TA \\
\hline Análise 1 & 0,131 & 0,009 & 0,217 \\
Análise 2 & 0,068 & 0,003 & 0,129 \\
\hline
\end{tabular}

TR - resistências elétricas; TP - piso aquecido; TA - sistema de aquecimento por convecção.

Apesar de os animais nas instalações com TA terem ganho mais peso que os da instalação com TP, o consumo específico de TA foi o maior (e, portanto, pior) que o TP. Assim, sob o aspecto consumo específico, a instalação com TP foi o melhor sistema e TA foi o pior dos três sistemas, sendo que TP apresentou consumo específico de aproximadamente 4,15\% de TA e de $6,87 \%$ de TR, durante a análise 1, e 2,32\% de TP e 4,41\% de TR, durante a análise 2.

A quantidade de energia elétrica consumida para o aumento de $1^{\circ} \mathrm{C}$ em $1 \mathrm{~m}^{3}$ de ar nesta edificação está apresentada na Tabela 6. Estes dados são locais e são válidos para o tipo de edificação aqui estudada.

TABELA 6. Quantidade de energia consumida $(\mathrm{kWh})$ por diferentes sistemas de aquecimento para leitões em creche, para o aumento de $1{ }^{\circ} \mathrm{C} \mathrm{m}^{-3}$ de ar na instalação. Total electrical consumption ( $\mathrm{kWh}$ ) by different heating systems for piglet nursery for increasing $1^{\circ} \mathrm{C}^{\mathrm{m}}{ }^{-3}$ of air in the facilities.

\begin{tabular}{cccc}
\hline & \multicolumn{3}{c}{ Quantidade de Energia Consumida $(\mathrm{kWh})$ para o Aumento de $1{ }^{\circ} \mathrm{C} \mathrm{m}^{-3} \mathrm{de} \mathrm{ar}$} \\
\cline { 2 - 4 } & $\mathrm{TR}$ & $\mathrm{TP}$ & $\mathrm{TA}$ \\
Análise 1 & 0,14 & 0,11 & 0,26 \\
Análise 2 & 0,26 & 0,15 & 0,43 \\
Média das análises & 0,20 & 0,13 & 0,35 \\
\hline
\end{tabular}

TR - resistências elétricas; TP - piso aquecido; TA - sistema de aquecimento por convecção

Comparando-se os resultados de energia consumida (kWh) para o aumento de $1^{\circ} \mathrm{C} \mathrm{m}^{-3} \mathrm{de}$ ar, observa-se que os maiores valores de energia consumida de $0,35 \mathrm{kWh}$ foram nas instalações com TA. O sistema mais eficiente, utilizando a análise do índice ( $\mathrm{kWh}$ para aumento de $1^{\circ} \mathrm{C} \mathrm{m}^{-3} \mathrm{de}$ ar) é o TP, pois utiliza a menor quantidade de energia para aumentar $1{ }^{\circ} \mathrm{C} \mathrm{m}^{-3} \mathrm{de}$ ar.

$\mathrm{Na}$ comparação entre as três tecnologias propostas, observa-se que as médias de temperatura de bulbo seco diferiram $(\mathrm{p}<0,05)$, quando os tratamentos foram comparados entre si e com o ambiente externo. As médias de temperatura de bulbo seco do ar estão apresentadas na Tabela 7.

Considerando a faixa de Tbs desejada $\left(28-30^{\circ} \mathrm{C}\right)$ e as médias de Tbs obtidas, nenhum dos tratamentos foi eficiente. Porém, foi constatado, nas duas análises, que todos os sistemas foram capazes de alcançar Tbs máximas de $30^{\circ} \mathrm{C}$. 
TABELA 7. Médias de temperatura de bulbo seco do ar $\left({ }^{\circ} \mathrm{C}\right)$ e ITU, obtidas em creche para leitões, utilizando diferentes sistemas de aquecimento. Mean values of dry-bulb temperature $\left({ }^{\circ} \mathrm{C}\right)$ and $\mathrm{THI}$, in piglet nursery, using different heating systems.

\begin{tabular}{lcccc}
\hline & \multicolumn{4}{c}{ Temperatura de Bulbo Seco do Ar $\left({ }^{\circ} \mathrm{C}\right)$ - Tbs } \\
\cline { 2 - 5 } Análise 1 & TR & TP & TA & Text \\
\cline { 2 - 4 } Análise 2 & $25,5( \pm 2,3)^{\mathrm{a}}$ & $24,2( \pm 5,8)^{\mathrm{c}}$ & $25,1( \pm 6,1)^{\mathrm{b}}$ & $18,8( \pm 4,2)^{\mathrm{d}}$ \\
& $26,2( \pm 2,4)^{\mathrm{a}}$ & $24,0( \pm 5,23)^{\mathrm{c}}$ & $25,3( \pm 2,6)^{\mathrm{b}}$ & $20,0( \pm 5,2)^{\mathrm{d}}$ \\
\cline { 2 - 5 } Análise 1 & \multicolumn{4}{c}{ Indice de Temperatura e Umidade $(\mathrm{ITU})$} \\
Análise 2 & $75( \pm 3)^{\mathrm{a}}$ & $73( \pm 3)^{\mathrm{c}}$ & $74( \pm 3)^{\mathrm{b}}$ & $66( \pm 5)^{\mathrm{d}}$ \\
an,b,c,d - Médias seguidas de letras diferentes na mesma linha diferem $(\mathrm{p}<0,05)$ pelo teste de Tukey. TR - resistências \\
elétricas; TP - piso aquecido; TA - sistema de aquecimento por convecção; Text - ambiente externo às salas.
\end{tabular}

Com relação à distribuição da Tbs ao longo das análises, é possível afirmar que TR manteve-se, na maior parte do tempo ( $42 \%$ dos dados analisados), entre 25 e $27^{\circ} \mathrm{C}$. O mesmo comportamento foi encontrado para TA, todavia, com porcentagem pouco menor $(40 \%)$. Porém, a Tbs de TP manteve-se entre 20 e $24{ }^{\circ} \mathrm{C}$, na maior parte do tempo (aproximadamente $49 \%$ ), sendo, portanto, o menos eficiente.

Considerando a faixa de temperatura utilizada nestas análises $\left(28\right.$ a $\left.30^{\circ} \mathrm{C}\right)$ e a faixa de umidade relativa dada pela literatura de 50 a 70\% (MOURA, 1999), o ITU ideal para leitões nesta fase é de 77 a 84. Todos os tratamentos foram diferentes estatisticamente entre si e quando comparados ao ambiente externo, e encontraram-se abaixo da zona de conforto dos animais, sendo TR o melhor e TP o pior tratamento neste quesito.

PANDORFI et al. (2004) testaram sistemas de aquecimento para leitões em maternidade. As tecnologias testadas foram: piso aquecido (ROSSI et al., 2005), lâmpada incandescente de 200W, resistências elétricas de $200 \mathrm{~W}$ e lâmpada infravermelha de $250 \mathrm{~W}$. Os autores encontraram que o piso térmico foi o mais eficiente nas trocas de calor sensível por condução, promovendo melhor condição de conforto para os animais. No entanto, o estudo foi realizado no interior de abrigos escamoteadores que possuíam um volume de $0,53 \mathrm{~m}^{3}$. O volume total da sala de creche a ser aquecido é de aproximadamente $80 \mathrm{~m}^{3}$. SOBESTIANSKY et al. (1987) comentaram que a utilização do escamoteador proporciona melhor acondicionamento ambiental aos leitões.

Desta forma, considerando a eficiência dos tratamentos com relação à temperatura de bulbo seco (do ar) recomendada para a fase de creche (BROWN-BRANDL et al., 2003), o aquecimento por resistências elétricas suspensas foi o melhor, seguido pelo aquecimento do ar por convecção. $\mathrm{O}$ aquecimento por piso aquecido foi o pior, considerando as médias de Tbs encontradas.

\section{CONCLUSÕES}

O sistema de aquecimento por piso (TP) foi melhor sob o aspecto de uso de energia elétrica, e o sistema de aquecimento por resistências elétricas suspensas (TR) foi o que melhor atendeu às necessidades térmicas dos animais.

\section{AGRADECIMENTOS}

À Fundação de Amparo à Pesquisa do Estado de São Paulo (FAPESP), pelo apoio financeiro ao trabalho, e ao CNPq, pelo apoio na forma de bolsa de doutorado.

\section{REFERÊNCIAS}

BESHADA, E.; ZHANG, Q.; BORIS, R.A.Y. A cost effective heating method for piglets in swine farrowing barns. In: CSBE/SCGAB 2006 ANNUAL CONFERENCE, 2006, Edmonton, Proceedings... Paper n.06-224 
BONETT, L.P.; MONTICELLI, C.J. Suínos: o produtor pergunta, a Embrapa responde. Coleção 500 Perguntas - 500 Respostas. 2.ed. Brasília: Embrapa - SPI, 1997. 243 p.

BROWN-BRANDL, T.M., NIENABER, J.A., XIN, H, GATES, R.S. A literature review of swine heat and moisture production. In: SWINE HOUSING, 2., 2003, North Carolina. Proceedings... p.31-40, (ASAE, 701P1303).

CONAB. COMPANHIA NACIONAL DE ABASTECIMENTO. Custo de produção de suínos por $U F$, 2008. Disponível em: <http://www.conab.gov.br>. Acesso em: 10 fev. 2009.

GIROTTO, A.F; TALAMINI, D.J.D. Administração da propriedade suinícola. In: SOBESTIANSKY, J. (Ed.). Suinocultura intensiva: Produção manejo e saúde do rebanho. Concórdia: EMBRAPA-CNPSA, 1998. p.292-298.

INCROPERA, F.P.; DEWITT, D.P. Fundamentos de transferência de calor e massa. EDIÇÃO. Rio de Janeiro: Livros Técnicos e Científicos Editora, 1998. p.262-266.

MERLOT, E.; MEUNIER-SALAÜN, M.; PRUNIER, A. Behavioural, endocrine and immune consequences of mixing in weaned piglets. Applied Animal Behaviour Sciences, Amsterdam, v.85, n.3-4, p.247-257, 2004.

MOURA, D.J. Ventilação na suinocultura. In: SILVA, I.J.O. Ambiência e qualidade na produção industrial de suínos. Piracicaba: FEALQ, 1999. p.149-179.

OLIVEIRA, C.E.L. de; GARCIA, J.L.; DE LA PLAZA, S.; CHAYA, C. Comparação do coeficiente global de perdas de calor para casa de vegetação aquecida usando diferentes técnicas para eficiência energética. Engenharia Agrícola, Jaboticabal, v.26, n.2, p.354-364, 2006.

PANDORFI, H.; SILVA, I.J.O.; MOURA, D.J.; SEVEGNANI, K.B. Análise de imagem aplicada ao estudo do comportamento de leitões em abrigo escamoteador. Engenharia Agrícola, Jaboticabal, v.24, n.2, p.274-284, 2004.

ROSSI, L.A.; CARDOSO, P.E.R.; BERALDO, A.L. Avaliação térmica de placas de argamassa de cimento e casca de arroz aquecidas por resistência elétrica. Engenharia Agrícola, Jaboticabal, v.25, n.1, p.37-45, 2005.

SARUBBI, J. Bem-estar dos animais e uso racional de energia elétrica em sistemas de aquecimento para leitões desmamados. 2009. 210 f. Tese (Doutorado em Engenharia Agrícola) Faculdade de Engenharia Agrícola, Universidade Estadual de Campinas, Campinas, 2009.

SOBESTIANSKY, J. PERDOMO, C.C.; OLIVEIRA, P.A.V. de; OLIVEIRA, J.A. Efeito de diferentes sistemas de aquecimento no desempenho de leitões. Concórdia: Embrapa Suínos e Aves, 1987, p.1-3. (Comunicado Técnico, 122).

TALAMINI, D.J.D. ; MARTINS, F.M.; ARBOIT, C., WOLOZSYN, N. Custos agregados da produção integrada de suínos nas fases de leitões e de terminação. Custos e agronegócios online, v.2, out. 2006. Edição especial. Disponível em: <http://custoseagrpneciosonline.com.br>. Acesso em: 8 set. 2010

WOLTER, B.F.; ELLIS, M.; CURTIS, S.E. Group size and floor-space allowance can affect weanling-pig performance. Journal of Animal Science, Champaign, v.78, p.2.062-2.067. 2000.

XIN, H.; ZHOU, H.; BUNDY, D.S. Comparison of energy use and piglet performance between conventional and energy-efficient heat lamps. Applied Engineering in Agriculture, St. Joseph, v.13, n.1, p.95-99, 1997.

XIN, H.; ZHANG, Q. Preference for lamp or mat heat by piglets at cool and warm ambient temperatures with low to high drafts. Applied Engineering in Agriculture, St. Joseph, v.15, n.5, p.547-551, 1999. 